\title{
Beyond the Standard Model searches at HERA
}

\author{
Oleksii Turkot* (on behalf of the $\mathrm{H} 1$ and ZEUS Collaborations) \\ DESY \\ E-mail: oleksii.turkotecern.ch
}

\begin{abstract}
The search for physics beyond the Standard Model was pursued at the HERA ep collider, both for dedicated channels and general searches using the data on deep inelastic scattering. The high precision and wide kinematic range of the data collected by the H1 and ZEUS experiments not only allowed determination of the parton distribution functions (PDFs) from HERA data only, but also for simultaneous fits to be performed of PDFs with parameters of models beyond the Standard Model (BSM), providing an unbiased search for "new physics". Such a final simultaneous analysis was performed in the framework of eeqq contact interactions, including leptoquarks, and BSM modifications of the weak couplings. No statistically significant deviation from the Standard Model was observed and the most stringent limits from HERA on the considered models were set. Limits on the compositeness scale of general contact interactions and mass scale of the heavy-leptoquark scenarios were obtained in the $\mathrm{TeV}$ range.
\end{abstract}

XXIX International Symposium on Lepton Photon Interactions at High Energies - LeptonPhoton2019 August 5-10, 2019

Toronto, Canada

${ }^{*}$ Speaker. 


\section{Introduction}

The Standard Model of particle physics provides a successful description of almost all current experimental data. Still, it does not include some of the experimentally observed phenomena, such as dark matter and neutrino masses. Therefore practically every high energy experiment include searches for the evidence of beyond the Standard Model (BSM) physics in its analysis program. The experiments at HERA, the electron ${ }^{1}$-proton collider which operated in 1992-2007 with an electron beam energy of $E_{e} \simeq 27.5 \mathrm{GeV}$ and proton beam energies of $E_{p} \simeq 920,820,575$ and $460 \mathrm{GeV}$, were no exception. Beyond the Standard Model searches at HERA include, but are not limited to, searches for pentaquark states, studies of events with isolated or multiple highmomentum leptons, and BSM searches combined with QCD analyses.

\section{Searches for the pentaquark states}

In a 1997 paper [1], Diakonov, Petrov and Polyakov proposed a pentaquark baryon state consisting of $u, d$, and $s$ quarks, and in 2003 first evidence of a suitable narrow baryon resonance with a mass of approximately $1530 \mathrm{MeV}$ was reported by LEPS experiment [2], followed by similar $\sim 4 \sigma$ evidence reports from SAPHIR and CLAS experiments. This baryon resonance state was interpreted as an exotic $\Theta^{+}(u u d d \bar{s})$ pentaquark baryon. In $2004 \Theta^{+}$evidence was reported by HERMES [3] and ZEUS [4] experiments at HERA, and in the Review of Particle Physics [5] $\Theta^{+}$ was stated as established, although not yet fully studied, resonance.

At the same time the HERA-B collaboration published [6] search results with no evidence of a baryon resonance near $1530 \mathrm{MeV}$ and set limits on $\Theta^{+}$production. Similar limits were set by the H1 collaboration [7] in 2006, and in 2016, after analysing 2003-2007 data, ZEUS collaboration published results [8] with no evidence of a narrow baryonic state and limits on its production cross section were set. The distributions of the $K_{S}^{0} p(\bar{p})$ invariant mass published by $\mathrm{H} 1$ in 2006 and ZEUS in 2016 are shown in Fig. 1. Taking into account results from several other experiments it was concluded [9] that original results with evidence of $u u d d \bar{s}$ pentaquark state had underestimated backgrounds and biased selection criteria.

For completeness it is worth noting that $c \bar{c} u u d$ pentaquark states were discovered at the LHCb experiment in 2015 [10] and observed in three states with 5-7 $\sigma$ significance [11].

\section{Isolated and multi-leptons searches}

A combined analysis [12] of events containing an isolated high transverse momentum $\left(P_{T}\right)$ electron or muon and missing transverse momentum was performed in the common phase space on data collected by the $\mathrm{H} 1$ and ZEUS detectors. In the Standard Model such events dominantly result from single $W$ boson production, and an event excess could be a good indication of BSM physics. At large hadronic transverse momentum $P_{T}^{X}>25 \mathrm{GeV}, 23$ events were observed in the $e^{+} p$ data, as shown in Fig. 2 (left), while the Standard Model prediction is $14.0 \pm 1.9$.

A similar procedure of the bin-by-bin combined analysis was used in a search for events with at least two high- $P_{T}$ leptons. Within the Standard Model the production of multi-lepton final states

\footnotetext{
${ }^{1}$ Here and afterwards "electron" refers to both electrons and positrons, unless otherwise stated.
} 

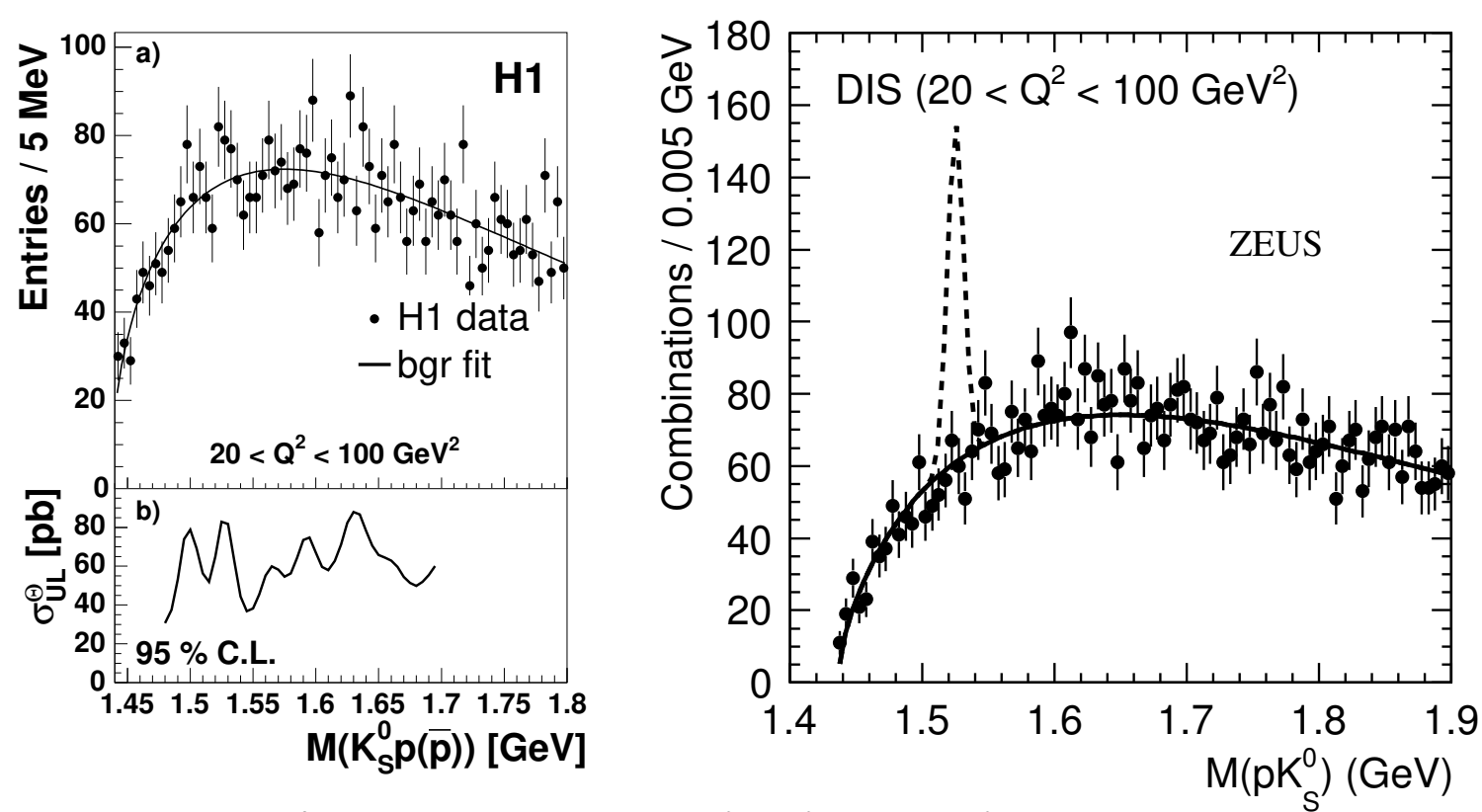

Figure 1: Invariant $K_{S}^{0} p(\bar{p})$ mass spectra for $20 \mathrm{GeV}^{2}<Q^{2}<100 \mathrm{GeV}^{2}$ measured in 1996-2000 by H1 [7] (left) and in 2002-2007 by ZEUS [8] (right) experiments. The dashed line on the right figure represents the signal corresponding to the previous ZEUS result measured on data taken in 1996-2000 [4].
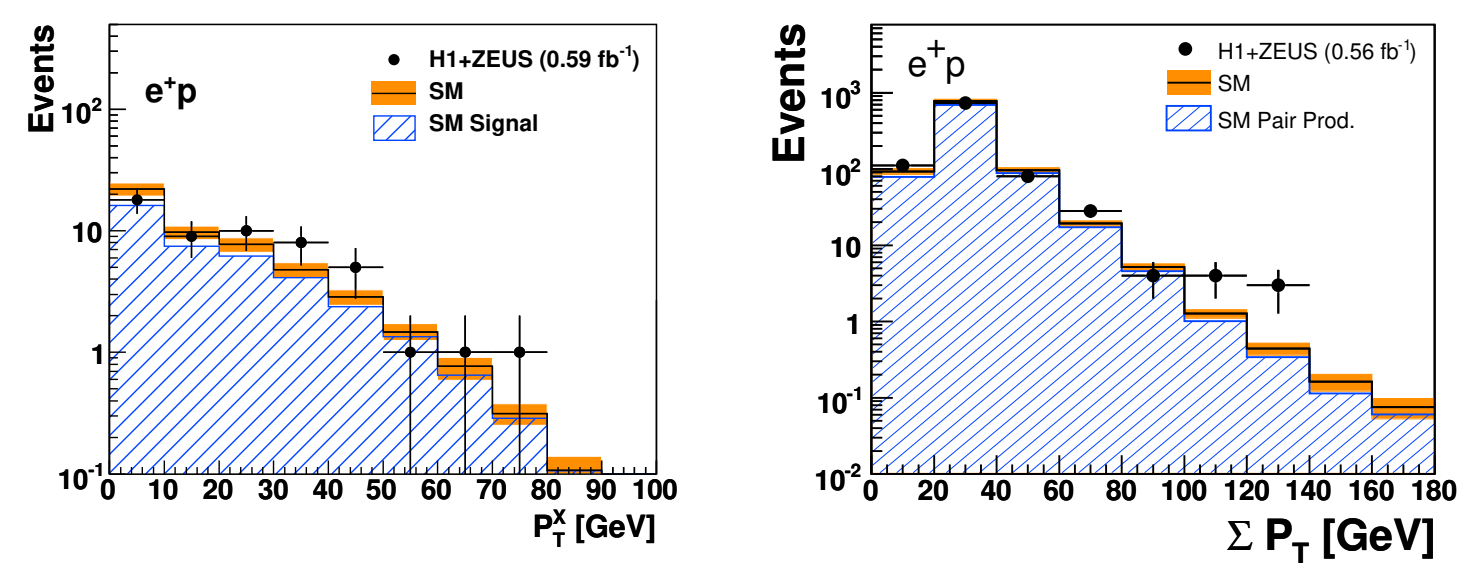

Figure 2: The distribution of the hadronic transverse momentum $P_{T}^{X}$ of events with an isolated electron or muon and missing transverse momentum (left) and distribution of the scalar sum of the transverse momentum $\sum P_{T}$ for combined di-lepton and tri-lepton event topologies (right) for the $e^{+} p$ HERA data. 
in $e^{ \pm} p$ collisions proceeds mainly via photon-photon interactions with low cross section. In total, no events with a scalar sum of transverse momenta of all leptons $\sum P_{T}>100 \mathrm{GeV}$ were observed in $e^{-} p$ data, and seven such events were observed in $e^{+} p$ data, displayed in Fig. 2 (right), while the Standard Model expectations are $1.19 \pm 0.12$ and $1.94 \pm 0.17$, respectively.

\section{QCD analyses combined with the BSM searches}

Deep-inelastic $e^{ \pm} p$ scattering (DIS) data collected by the H1 and ZEUS experiments span six orders of magnitude in negative four-momentum-transfer squared, $Q^{2}$, and Bjorken $x$, and provide a unique opportunity to determine the parton distribution functions (PDFs) with data from a single experiment. The high precision of the $0.5 \mathrm{fb}^{-1}$ of collected data and longitudinal polarisation of the lepton beams during 2002-2007 data taking allowed the H1 collaboration not only to determine electroweak parameters together with the PDF parameters, but also to study possible BSM modifications of the weak couplings in the same combined procedure [14]. Limits on the potential modifications of the weak coupling parameters due to the BSM physics evaluated at $68 \%$ C.L. are shown in Fig. 3 (left). Also, for the first time, a possible scale dependence of the weak coupling parameters was studied in the range $12 \mathrm{GeV}<\sqrt{Q^{2}}<100 \mathrm{GeV}$, and no significant deviation from the Standard Model expectation was observed [14].
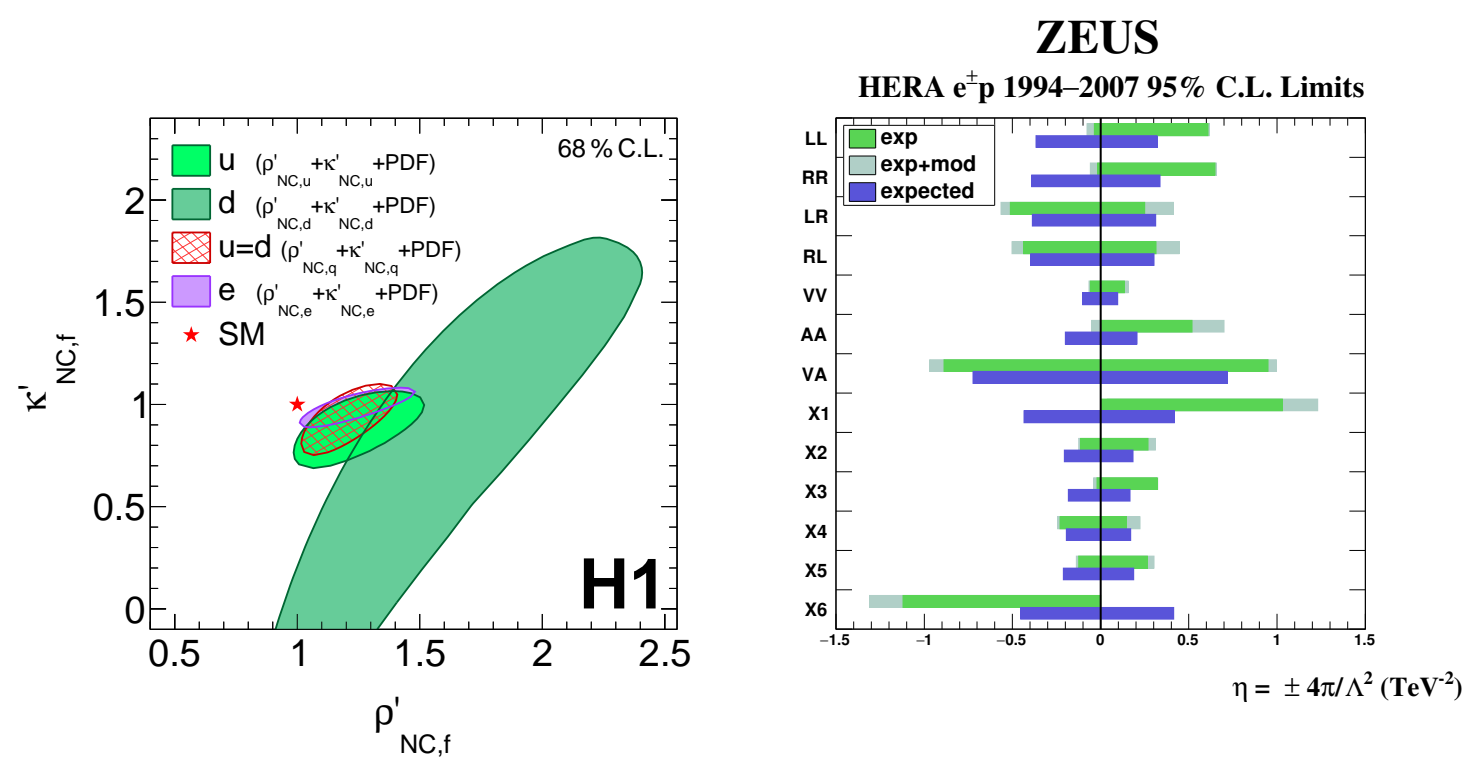

Figure 3: Results for the weak couplings BSM modification parameters $\rho_{\mathrm{NC}, f}^{\prime}$ and $\kappa_{\mathrm{NC}, f}^{\prime}$ for $u$ - and $d$-type quarks and electrons at $68 \%$ C.L. (left) and 95\% C.L. limits on the contact interaction coupling strength $\eta= \pm 4 \pi / \Lambda^{2}$ (right) obtained in the procedure combined with the QCD analysis.

All inclusive neutral and charged current $e^{ \pm} p$ DIS cross sections published by the H1 and ZEUS collaborations for zero beam polarisation were combined into one coherent data set [15] corresponding to a luminosity of around $1 \mathrm{fb}^{-1}$. These data were used to search for deviations from the Standard Model predictions in the framework of eeqq contact interactions (CI) [16]. To avoid any possible bias raleted to the choice of PDF set the analysis procedure was based on a 
simultaneous fit of PDF parameters and the CI coupling. Limits on the CI coupling were set for several general CI models, displayed in Fig. 3 (right), and scenarios with heavy leptoquarks. For some of the general CI and heavy leptoquark models simultaneous CI and PDF fits provide improved description of the combined HERA inclusive data, with the difference from the Standard Model predictions at the level of 3-4 $\sigma$.

\section{References}

[1] D. Diakonov, V. Petrov and M.V. Polyakov, Z. Phys. A 359, 305 (1997);

[2] LEPS Collaboration, T. Nakano et al., Phys. Rev. Lett. 91, 012002 (2003);

[3] HERMES Collaboration, A. Airapetian et al., Phys. Lett. B 585, 213 (2004);

[4] ZEUS Collaboration, S. Chekanov et al., Phys. Lett. B 591, 7 (2004);

[5] Particle Data Group, S. Eidelman et al., Phys. Lett. B 592, 1 (2004);

[6] HERA-B Collaboration, I. Abt et al., Phys. Rev. Lett. 93, 212003 (2004);

[7] H1 Collaboration, A. Aktas et al., Phys. Lett. B 639, 202 (2006);

[8] ZEUS Collaboration, H. Abramowicz et al., Phys. Lett. B 759, 446 (2016);

[9] Particle Data Group, W. M. Yao et al., J. Phys. G 33, 1 (2006);

[10] LHCb Collaboration, R. Aaij et al., Phys. Rev. Lett. 115, 072001 (2015);

[11] LHCb Collaboration, R. Aaij et al., Phys. Rev. Lett. 122, 222001 (2019);

[12] H1 and ZEUS Collaborations, F. D. Aaron et al., JHEP 1003, 035 (2009);

[13] H1 and ZEUS Collaborations, F. D. Aaron et al., JHEP 0910, 013 (2009);

[14] H1 Collaboration, V. Andreev et al., Eur. Phys. J. C 78, 777 (2018);

[15] H1 and ZEUS Collaborations, H. Abramowicz et al., Eur. Phys. J. C 75, 580 (2015);

[16] ZEUS Collaboration, H. Abramowicz et al., Phys. Rev. D 99, 092006 (2019). 